\title{
Relativistic calculations of secondary ions produced in plasma desorption mass spectrometry
}

\author{
R.M. Yoosoofmiya *, K.A.I.L.W. Gamalath \\ Department of Physics, University of Colombo
}

\begin{abstract}
In Time of Flight (TOF) mass spectrometry, the ratio; mass/charge of ions of the sample under investigation is determined by a relationship between time of flight and spectrometer parameters. Usually this relationship is derived using classical (Newtonian) mechanics. Since high energies are involved in the flight of the particles a relativistic treatment is considered. It is investigated whether the relativistic effects are significant or not, for the motion of secondary ions produced in the Plasma Desorption Mass Spectrometer. Treating the motion of these ions relativistically, a relationship between the Time of Flight (TOF) and mass/charge of the ion was derived. Using advanced mathematical software package Maple 6, the derived relationship was compared with classically obtained results. Then it was found that relativistic effects are not significant and they can be ignored for the linear Plasma Desorption Mass Spectrometer.
\end{abstract}

\section{INTRODUCTION}

The objective of the study is to investigate whether there are any significant relativistic effects in the motion of secondary ions produced in the Plasma Desorption Mass Spectrometer (PDMS).

Plasma desorption mass spectrometry is developed by combining electronic sputtering and time of flight method. Sputtering can be loosely described as the process of ejection of molecules from a surface when it is bombarded with some energy source. In Time of Flight mass spectrometry method, ions of molecules whose mass is to be determined, are needed to be produced. This can be achieved by bombarding the sample by some energy source so that sputtering occurs. Thus sputtered ions are termed as "secondary ions" produced in the plasma desorption mass spectrometry.

This new method of mass spectrometry was created by Macfarlane and his coworkers. This was following the discovery in 1974 of fission fragments from $\mathrm{Cf}^{252}$ source can sputter and ionize intact organic molecules from a surface ${ }^{6}$. Although this

\footnotetext{
* Corresponding Author
} 
process, at the time was named as Plasma Desorption, a more appropriate name would be secondary ion mass spectrometry (SIMS) with fast ion primary particles and TOF mass analysis ${ }^{6}$.

\section{PLASMA DESORPTION MASS SPECTROMETER}

A conventional Plasma Desorption Mass Spectrometer is represented schematically in figure 1. The sample under investigation is prepared as a smooth thin disc. Californium252 is used as the energy source to sputter the sample. When Cf252 undergoes nuclear decay, it emits two fission fragments which are almost collinear. One of these fragments is used to sputter the sample and other one is used to mark the starting of the event of sputtering, with a start pulse ${ }^{6}$.

When an ion is ejected (sputtered) from the surface of the sample, it undergoes acceleration under the potential difference $V_{l}$. When the ion passes through the first grid, it enters a field free region, and flies with a uniform velocity. When the ion enters the second acceleration region, again it is accelerated under a potential difference $V_{2}$, to increase the detection efficiency. When the ion hits the stop detector it generates a stop pulse. The time difference between the start and the stop pulses is a measure of the time of flight of the ion concerned. The start and stop pulses are then fed in to a Time to Digital Converter (TDC). The flight times are then recorded in a computer based multi channel analyzer (MCA). The time to digital converter has a resolution of 1 nanosecond ${ }^{6}$.
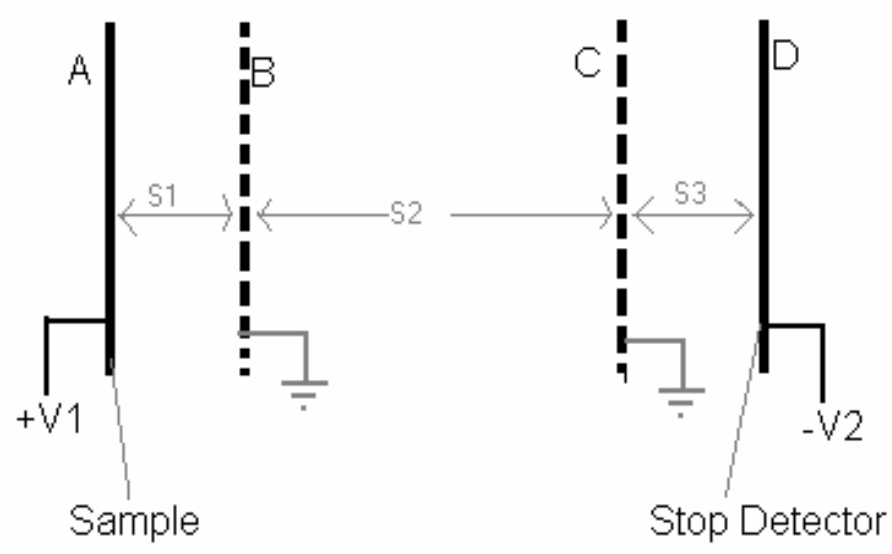

Figure 1. Simplified Schematic Diagram

A mathematical relationship between the flight time and the mass/charge of an ion, can be easily obtained by treating the motion of the ion described above, using classical mechanics ${ }^{6}$. 
Equation for Time-of-Flight $(T)$ is given below.

$$
T=\sqrt{\frac{2 m}{q}}\left\{\frac{S_{1}}{V_{1}}\left(\sqrt{V_{0}+V_{1}}-\sqrt{V_{0}}\right)+\frac{S_{2}}{2 \sqrt{V_{0}+V_{1}}}+\frac{S_{3}}{V_{2}}\left(\sqrt{V_{0}+V_{1}+V_{2}}-\sqrt{V_{0}+V_{1}}\right)\right\}
$$

The measured Time becomes: $T_{m}=T+T_{0}$ where $T_{0}$ is the delay in the circuit. Thus the relationship between the measured time $T_{m}$ and $m / q$ can be summarized as;

$$
T_{m}=A \sqrt{m / q}+T_{0}
$$

where $A$ is a constant.

By using equation (2), mass/charge can be found if the spectrometer parameters are known.

Spectrometer parameters used in the equations are defined as follows. $m$ and $q$ stands for mass and charge of the ion respectively. The corresponding voltage $V_{0}$ is defined as $q V_{0}$ being the kinetic energy due to initial axial velocity. Voltages $V_{1}$ and $V_{2}$ are the acceleration voltages in region $\mathrm{A}$ to $\mathrm{B}$ and region $\mathrm{C}$ to $\mathrm{D}$ respectively. $S_{1}, S_{2}$ and $S_{3}$ are the distances between A-B, B-C and C-D respectively. $v_{1}$ and $v_{2}$ stands for velocity at B and D respectively.

\section{RELATIVISTIC TREATMENT OF THE SECONDARY IONS}

The sputtered ions from the sample, in the PDMS, undergo acceleration voltage $V_{l}$, which is typically of the order $10^{4}$ volts. Therefore the ions can reach relativistic velocities. Since the time resolution of the PDMS at the Department of Physics is as high as 1 nanosecond ${ }^{2}$ at high velocities, classical approximation of motion may be inadequate. Thus it is worthwhile to see if the relativistic effects in the motion of secondary ions could be detected.

When treating the motion of secondary ions, the mass spectrometer (and the lab in which it is installed in) is taken as the rest frame. The motion of the ions is considered relative to this lab-frame. Kinetic energy and the momentum $p$, of the ions are taken as given by the following relativistic equations.

$$
\begin{aligned}
& \boldsymbol{p}=m_{0} \gamma \boldsymbol{u} \\
& K . E=m c^{2}-m_{0} c^{2}=m_{0} c^{2}(\gamma-1)
\end{aligned}
$$

Since the horizontal acceleration is much greater than the gravitational acceleration and vertical initial velocity, it was assumed in the derivation that the vertical motion of an ions is negligible. 
Consider the schematic diagram given in the Figure 1 of the PDMS again to derive relativistic expression. Let the initial axial velocity at $\mathrm{A}$ be $v_{0}$, and let the corresponding potential $V_{0}$ be defined by the following equation.

$$
q V_{0}=m_{0} c^{2}\left(\gamma\left(v_{0}\right)-1\right) \quad \text { where } \gamma\left(v_{0}\right)=\frac{1}{\sqrt{1-\frac{v_{0}}{c^{2}}}}
$$

this leads to the equation

$$
v_{0}=c \sqrt{1-\frac{1}{\left(1+\frac{q V_{0}}{m_{0} c^{2}}\right)^{2}}}
$$

Then the kinetic energy at B can be written as

$$
q\left(V_{0}+V_{1}\right)=m_{0} c^{2}\left(\gamma\left(v_{1}\right)-1\right)
$$

and by solving this for $V_{l}$ results in

$$
v_{1}=c \sqrt{1-\frac{1}{\left(1+\frac{q\left(V_{0}+V_{1}\right)}{m_{0} c^{2}}\right)^{2}}}
$$

Similarly velocity at $\mathrm{D}$ can be found to be given by

$$
v_{2}=c \sqrt{1-\frac{1}{\left(1+\frac{q\left(V_{0}+V_{1}+V_{2}\right)}{m_{0} c^{2}}\right)^{2}}}
$$

By using these expressions for the velocities, time of flight in each region (shown in figure 1) of the secondary ion particle can be calculated.

The rate of change of relativistic momentum is equal to the force experienced by the particle. Thus following equation can be written.

$$
f=\frac{d}{d t}\left(\not m_{0} v\right)=q \frac{V_{1}}{S_{1}}
$$

Integrating equation (8) from A to B leads to a expression for $t_{l}$.

$$
\int_{A}^{B} d t=\int_{A}^{B} \frac{m_{0} S_{1}}{V_{1} q} d(\mathcal{W})
$$




$$
t_{1}=\frac{m_{0} S_{1}}{q V_{1}}\left[v_{1} \gamma_{1}-v_{0} \gamma_{0}\right]
$$

$$
\text { where } \gamma_{i}=\frac{1}{\sqrt{1-\frac{v_{i}^{2}}{c^{2}}}}
$$

Similarly for region $\mathrm{C}$ to $\mathrm{D}$ :

$$
t_{3}=\frac{m_{0} S_{3}}{q V_{2}}\left[v_{2} \gamma_{2}-v_{1} \gamma_{1}\right]
$$

For Field free region B to C:

$$
t_{2}=\frac{S_{2}}{v_{1}}
$$

After expanding and simplifying equations (9) and (10) following expressions for $t_{1}$ and $t_{2}$ are obtained.

$$
\begin{aligned}
& t_{1}=\frac{S_{1} c}{V_{1}} \frac{m_{0}}{q}\left\{\sqrt{\left(1+\frac{V_{0}+V_{1}}{c^{2}} \frac{1}{m_{0} / q}\right)^{2}-1}-\sqrt{\left(1+\frac{V_{0}}{c^{2}} \frac{1}{m_{0} / q}\right)^{2}-1}-\sqrt{\left(1+\frac{V_{0}+V_{1}}{c^{2}} \frac{1}{m_{0} / q}\right)^{2}-1}-1\right. \\
& t_{3}=\frac{S_{3} c}{V_{2}} \frac{m_{0}}{q}\left\{\sqrt{\left(1+\frac{V_{0}+V_{1}+V_{2}}{c^{2}} \frac{1}{m_{0} / q}\right)^{2}}-1\right.
\end{aligned}
$$

$t_{2}$ can also be expressed in terms of potential, by substituting the expression for velocity $v_{2}$ given by equation (7) in to the equation (11). This leads to

$$
t_{2}=\frac{S_{2}}{c} \frac{1}{\sqrt{1-\frac{1}{\left(1+\frac{V_{0}+V_{1}}{c^{2}} \frac{1}{m_{0} / q}\right)^{2}}}}
$$


Finally, by adding equations (12), (13) and (14), Time of Flight (TOF) can be found.

$$
\begin{array}{r}
T_{\text {TOF }}=\frac{S_{1} c}{V_{1}} \frac{m_{0}}{q}\left\{\sqrt{\left(1+\frac{V_{0}+V_{1}}{c^{2}} \frac{1}{m_{0} / q}\right)^{2}}-1-\sqrt{\left(1+\frac{V_{0}}{c^{2}} \frac{1}{m_{0} / q}\right)^{2}}-1\right\}+\frac{S_{2}}{c} \frac{1}{\sqrt{\left(1-\frac{V_{0}+V_{1}}{c^{2}} \frac{1}{m_{0} / q}\right)^{2}}}+ \\
\frac{S_{3} c \frac{m_{0}}{V_{2}}}{q}\left\{\sqrt{\left(1+\frac{V_{0}+V_{1}+V_{2}}{c^{2}} \frac{1}{m_{0} / q}\right)^{2}-1-\sqrt{\left(1+\frac{V_{0}+V_{1}}{c^{2}} \frac{1}{m_{0} / q}\right)^{2}}-1}\right)^{\left(1+\frac{1}{q}\right)}
\end{array}
$$

Unlike the motion considered under Newtonian classical mechanics, relativistic motion tends to get saturated in terms of velocity, asymptotically approaching velocity of light rather then increasing indefinitely. Therefore it is expected that relativistic time of flight given by equation (15), to be greater than the classical time of flight given by the equation (1). This difference in Time of Flights is expected to increase as the parameters which tend to increase the velocity of the particle, increases.

Thus parameters such as S1, S2 and V1 shall be important in finding the significance of relativistic treatment of motion of secondary ions.

\section{ERROR OF MASS}

Consider the mass/charge of the secondary ion as a function as shown in the following equation.

$$
m / q=f\left(S_{1}, S_{2}, S_{3}, V_{0}, V_{1}, V_{2}, T_{0}, T_{T O F}\right)
$$

The error in $m / q$, can be found as;

$$
\begin{gathered}
\delta(m / q)^{2}=\left(\frac{\partial f}{\partial S_{1}}\right)^{2} \delta S_{1}^{2}+\left(\frac{\partial f}{\partial S_{2}}\right)^{2} \delta S_{2}{ }^{2}+\left(\frac{\partial f}{\partial S_{3}}\right)^{2} \delta S_{3}{ }^{2}+\left(\frac{\partial f}{\partial V_{0}}\right)^{2} \delta V_{0}^{2}+\left(\frac{\partial f}{\partial V_{1}}\right)^{2} \delta V_{1}^{2}+ \\
\left(\frac{\partial f}{\partial V_{2}}\right)^{2} \delta V_{2}^{2}+\left(\frac{\partial f}{\partial T_{0}}\right)^{2} \delta T_{0}^{2}+\left(\frac{\partial f}{\partial T_{T O F}}\right)^{2} \delta T_{T O F}{ }^{2}
\end{gathered}
$$


Equation (17) can be equivalently written as

$$
\begin{array}{r}
\delta(m / q)^{2}=\left(\frac{\partial T_{T O F}}{\partial S_{1}}\right)^{2} \delta S_{1}^{2}+\left(\frac{\partial T_{T O F}}{\partial S_{2}}\right)^{2} \delta S_{2}^{2}+\left(\frac{\partial T_{T O F}}{\partial S_{3}}\right)^{2} \delta S_{3}^{2}+\left(\frac{\partial T_{T O F}}{\partial V_{0}}\right)^{2} \delta V_{0}^{2}+\left(\frac{\partial T_{T O F}}{\partial V_{1}}\right)^{2} \delta V_{1}^{2}+ \\
\left(\frac{\partial T_{T O F}}{\partial V_{2}}\right)^{2} \delta V_{2}^{2}+\left(\frac{\partial T_{T O F}}{\partial T_{0}}\right)^{2} \delta T_{0}{ }^{2}+\delta T_{T O F}{ }^{2} /\left(\frac{\partial T_{T O F}}{\partial m}\right)^{2}
\end{array}
$$

By substituting appropriate values in to the above equation, the error of $\mathrm{m} / \mathrm{q}$ can be found.

\section{CALCULATIONS AND ANALYSIS}

The relativistic equation (15) which is used throughout this project happens to be non-linear. This equation is basically used for calculating the Time of Flight for given values of spectrometer parameters, or for finding the mass/charge for a given Time of Flight. For calculations in both of these cases, basically two options were available. One was, writing a computer program by using some high level language and the second, using a standard mathematical software package.

Maple 6 was chosen to do the calculations in the project due to high accuracy it offers and simplicity of use. Maple achieves most of its power from tightly integrated suite of symbolic and NAG's " numerical solvers. The new technology found in Maple 6 has application for scientific and engineering users in industry, research, and education ${ }^{3}$.

\section{Spectrometer parameters:}

Typical values for the spectrometer parameters ${ }^{6}$ are listed in table 1 . Unless otherwise stated, values for each of the parameters (Refer the figure 1) shall be taken as below.

Table 1. Typical values for the spectrometer parameters

\begin{tabular}{|l|l|}
\hline A to B distance & $\mathrm{S}_{1}=0.45 \mathrm{~cm}$ \\
\hline B to C distance & $\mathrm{S}_{2}=16 \mathrm{~cm}$ \\
\hline C to D distance & $\mathrm{S}_{3}=1.23 \mathrm{~cm}$ \\
\hline Initial axial potential & $\mathrm{V}_{0}=0$ volts \\
\hline Acceleration voltage (A to B) & $\mathrm{V}_{1}=10^{4}$ volts \\
\hline Acceleration voltage (C to D) & $\mathrm{V}_{2}=2500$ volts \\
\hline Velocity of light & $\mathrm{c}=299792458 \mathrm{~ms}^{-1}$ \\
\hline
\end{tabular}

\footnotetext{
* Numerical Algorithms Group
} 


\section{Preview of Times of Flight under Classical and Relativistic Models}

The curves of Time of Flight versus mass/charge of the secondary ions shall be plotted using the equations (1) and (14), which gives classical and relativistic Times of Flight respectively. In these plotting, only the TOF is considered without the offset time.

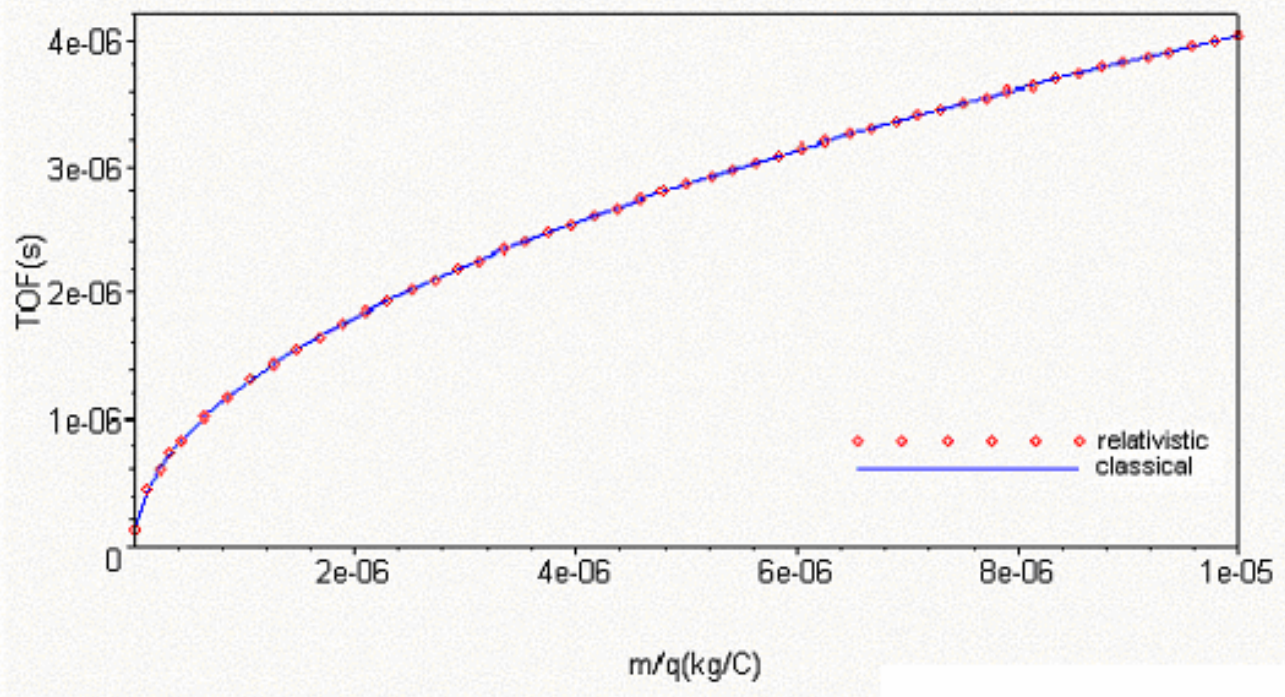

Figure 2. Times of Flight Vs mass/charge of secondary ion

This curve in the graph 1 is plotted in the mass/charge range of 1 a.m.u./e $\left(1.66 \times 10^{-27} / 1.602 \times 10^{-19} \mathrm{~kg} / \mathrm{C}\right)$ to 1000 a.m.u/e. It is seen that the relativistic time of flight is followed closely by the classical Time of Flight. It can be seen that for both models the Time of Flight is of the order $10^{-6}$ seconds. However the problem at hand requires looking in to the difference between the Flight times predicted by the two models, rather than further investigating the flight times.

\section{Calculation of Mass}

For a given Time of Flight and other mass-spectrometer parameters, $m / q$ value of the ions can be calculated, both by using relativistic equation (15) and by using classical equation (1).

Results of calculations of relativistic and classical masses for Times of Flight are tabulated below. The other mass spectrometer parameters have the usual values, given earlier.

\footnotetext{
* e is taken as charge of an electron. Throughout the report this notation will be used.
} 
Table 2. Times of flight and corresponding masses

\begin{tabular}{|c|r|c|c|r|}
\hline \multirow{2}{*}{ TOF (s) } & \multicolumn{3}{|c|}{ Mass (a.m.u) } & \multirow{2}{*}{ \%Difference } \\
\cline { 2 - 4 } & Relativistic & Classical & Difference & \\
\hline $2 \times 10^{-7}$ & 2.366661193223 & 2.366676891852 & 0.000015698629 & 0.0006633 \\
\hline $3 \times 10^{-6}$ & 532.502284968256 & 532.502300666874 & 0.000015698618 & 0.0000029 \\
\hline $6 \times 10^{-6}$ & 2130.009186968880 & 2130.009202667498 & 0.000015698617 & 0.0000007 \\
\hline
\end{tabular}

From the results in Table 2, it is seen that the difference in masses when calculated using relativistic and classical relations decrease as the time of flight increases. This difference shows it self at fifth decimal place in units of a.m.u/e. Also this difference is seen to be decreasing. It can be concluded that the significance of the difference rapidly fades as the measured time of flight increase (i.e. for heavy secondary ions produced in PDMS). From the results tabulated above, it can also be concluded that, in the TOF range of order of $10^{-7}$ seconds to $10^{-6}$ seconds, relativistic effects are negligible.

As it is possible to get an idea about the significance or the lack of significance, of the relativistic corrections by considering the difference between the calculated masses, some inferences could also be made about the relativistic treatment by comparing the time of flights calculated with relativistic and non-relativistic models.

\section{Estimation of error in $\mathbf{m} / \mathbf{q}$}

The equation (18) is used in calculating the error of $\mathrm{m} / \mathrm{q}$. Values of spectrometer parameters, substituted in this equation are given below in table $3^{6}$.

Table 3. Typical Values and Errors of spectrometer parameters

\begin{tabular}{|l|l|l|}
\hline \multicolumn{1}{|c|}{ Parameter } & \multicolumn{1}{c|}{ value } & \multicolumn{1}{c|}{ Error } \\
\hline $\mathrm{V}_{0}$ & $1 \mathrm{v}$ & $.01 \mathrm{v}$ \\
\hline $\mathrm{V}_{1}$ & $10^{4} \mathrm{v}$ & $05 \mathrm{v}$ \\
\hline $\mathrm{V}_{2}$ & $2500 \mathrm{v}$ & $10 \mathrm{v}$ \\
\hline $\mathrm{S}_{1}$ & $0.45 \mathrm{~cm}$ & $0.01 \mathrm{~mm}$ \\
\hline $\mathrm{S}_{2}$ & $16 \mathrm{~cm}$ & $0.01 \mathrm{~mm}$ \\
\hline $\mathrm{S}_{3}$ & $1.23 \mathrm{~cm}$ & $0.01 \mathrm{~mm}$ \\
\hline $\mathrm{T}_{0}$ & & $0.1 \mathrm{~ns}$ \\
\hline $\mathrm{T}_{\text {TOF }}$ & & $0.1 \mathrm{~ns}$ \\
\hline
\end{tabular}

Using the values in table 3, errors for several $\mathrm{m} / \mathrm{q}$ values are calculated and tabulated in table 4. 
Table 4. Errors in $\mathrm{m} / \mathrm{q}$

\begin{tabular}{|r|r|r|r|r|}
\hline \multirow{2}{*}{$\begin{array}{c}\text { m/q } \\
\text { (a.m.u. / e) }\end{array}$} & \multicolumn{2}{|c|}{ Relativistic } & \multicolumn{2}{c|}{ Newtonian } \\
\cline { 2 - 5 } & $\begin{array}{c}\delta(\mathbf{m} / \mathbf{q}) \\
\text { (a.m.u./e) }\end{array}$ & \% error & $\begin{array}{c}\delta \text { (m/q) } \\
(\text { a.m.u./e) }\end{array}$ & \% error \\
\hline 1 & 0.0022 & 0.2194 & 0.0022 & 0.2194 \\
\hline 5 & 0.0051 & 0.1011 & 0.0051 & 0.1011 \\
\hline 10 & 0.0074 & 0.0741 & 0.0074 & 0.0741 \\
\hline 50 & 0.0206 & 0.0412 & 0.0206 & 0.0412 \\
\hline 100 & 0.0351 & 0.0350 & 0.0351 & 0.0350 \\
\hline 500 & 0.1458 & 0.0291 & 0.1458 & 0.0291 \\
\hline 1000 & 0.2834 & 0.0283 & 0.2834 & 0.0283 \\
\hline 5000 & 1.3831 & 0.0276 & 1.3831 & 0.0276 \\
\hline 10000 & 2.7575 & 0.0275 & 2.7575 & 0.0275 \\
\hline 50000 & 13.7533 & 0.0275 & 13.7533 & 0.0275 \\
\hline
\end{tabular}

From the results tabulated above, it is seen that the errors in estimation of $\mathrm{m} / \mathrm{q}$ using the relativistic model shows itself at most in the third decimal place. But it was found that the difference between the $\mathrm{m} / \mathrm{q}$ values found from classical and relativistic models show only at best in the fifth decimal place.

So it can be concluded that, instrumental errors are at least 100 times more significant than the errors due to not considering the relativistic effects. Also it is seen that the percentage error decreases as $\mathrm{m} / \mathrm{q}$ increases.

\section{Difference between Times of Flight}

Considering factors such as computational time and flexibility, calculation of timeof-flight for a given mass/charge is much efficient than calculation of $\mathrm{m} / \mathrm{q}$ for a given timeof-flight. Therefore, the time of flight shall be considered in the study to see, how the relativistic effect becomes prominent when the variation of the other parameters of the mass spectrometer is taken in to account. 


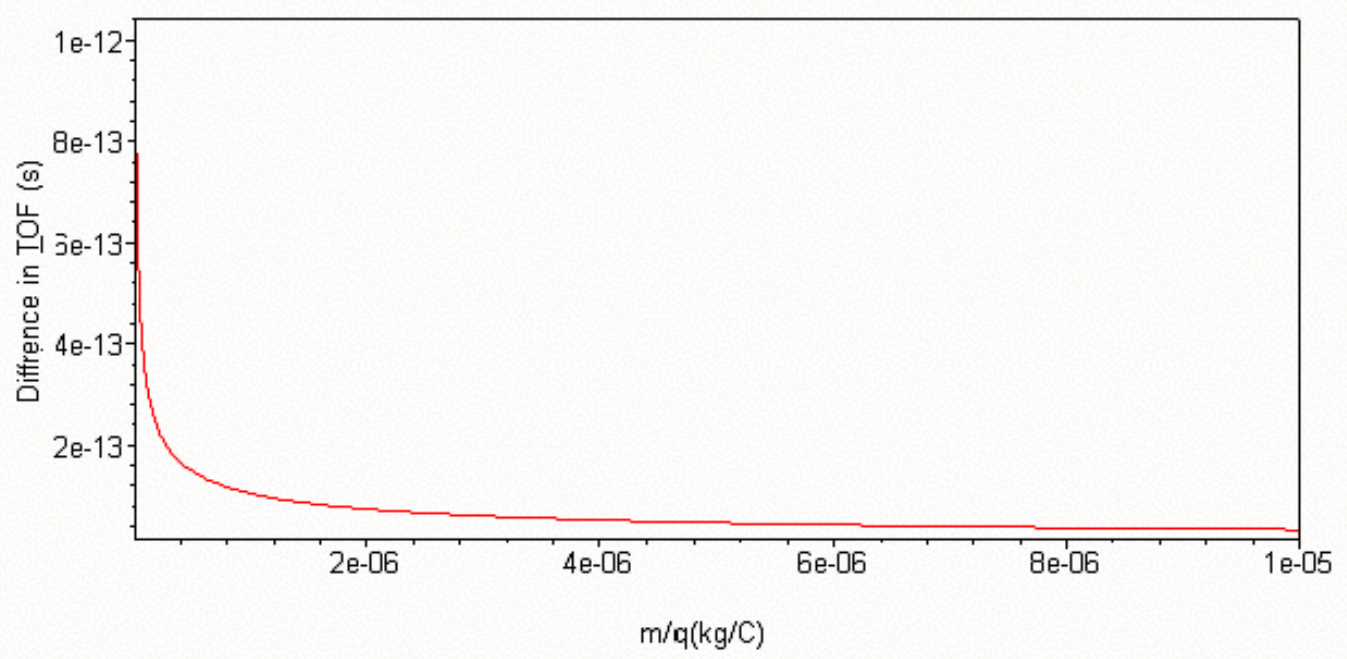

Figure 3. $\left(\mathrm{T}_{\text {rel }}-\mathrm{T}_{\text {cla }}\right)$ vs mass/charge

As before, the graph in figure 3 is plotted in the range of mass/charge from 1 a.m.u./e to 1000 a.m.u./e. As seen in the graph, the maximum difference between the calculated flight times under the two models is less than $10^{-12}$ seconds. The graph plotted here corresponds to the typical values of mass spectrometer parameters. For these values, it can be concluded that relativistic treatment does not give any significant improvement over accuracy, since the current time resolution of Plasma Desorption Mass Spectrometer at the department of physics is only 1 nanosecond.

As discussed earlier, an increase in spectrometer parameters such as $V_{1}, S_{2}$ and $S_{1}$ tends to pronounce the difference between the relativistic and classical results. The effect of these parameters shall be analyzed in what follows.

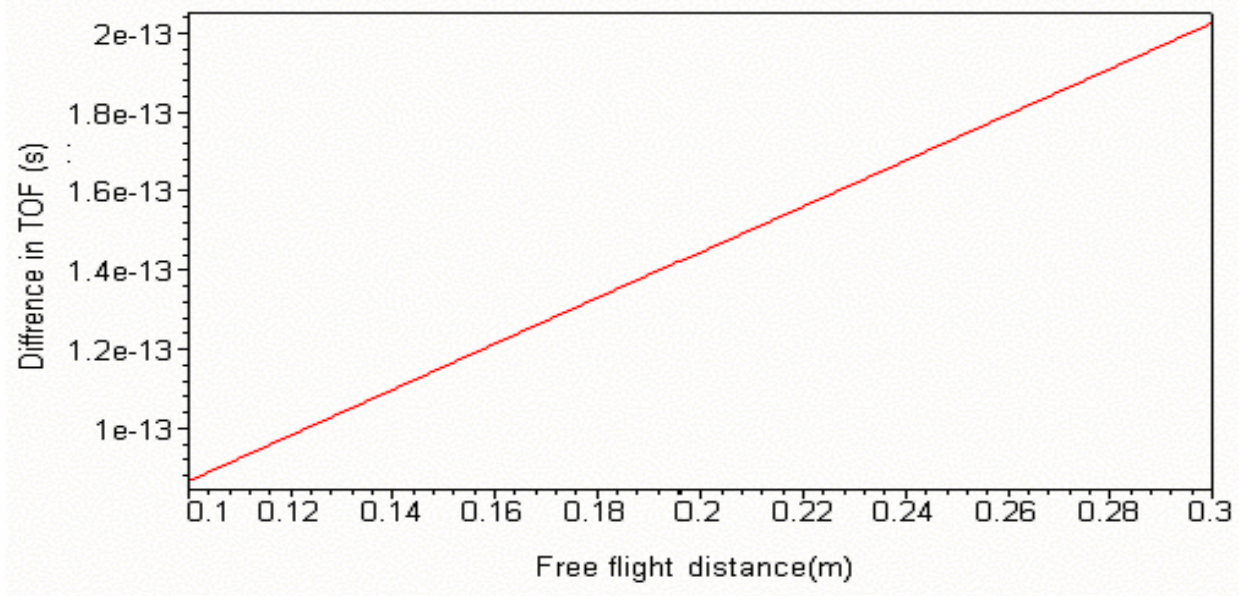

Figure 4. $\left(\mathrm{T}_{\text {rel }}-\mathrm{T}_{\text {cla }}\right)$ vs. free flight distance $\left(\mathrm{S}_{2}\right)$ 
The graph in figure 4 is plotted in the range of $S_{2}$, from $10 \mathrm{~cm}$ to $30 \mathrm{~cm}$. As can be seen, the difference in flight times is in the order of $10^{-13}$ seconds. The typical value of $S_{2}$, which is 16 centimeters, falls within this range. So it can be concluded that the relativistic effect is not important for the range of values considered.

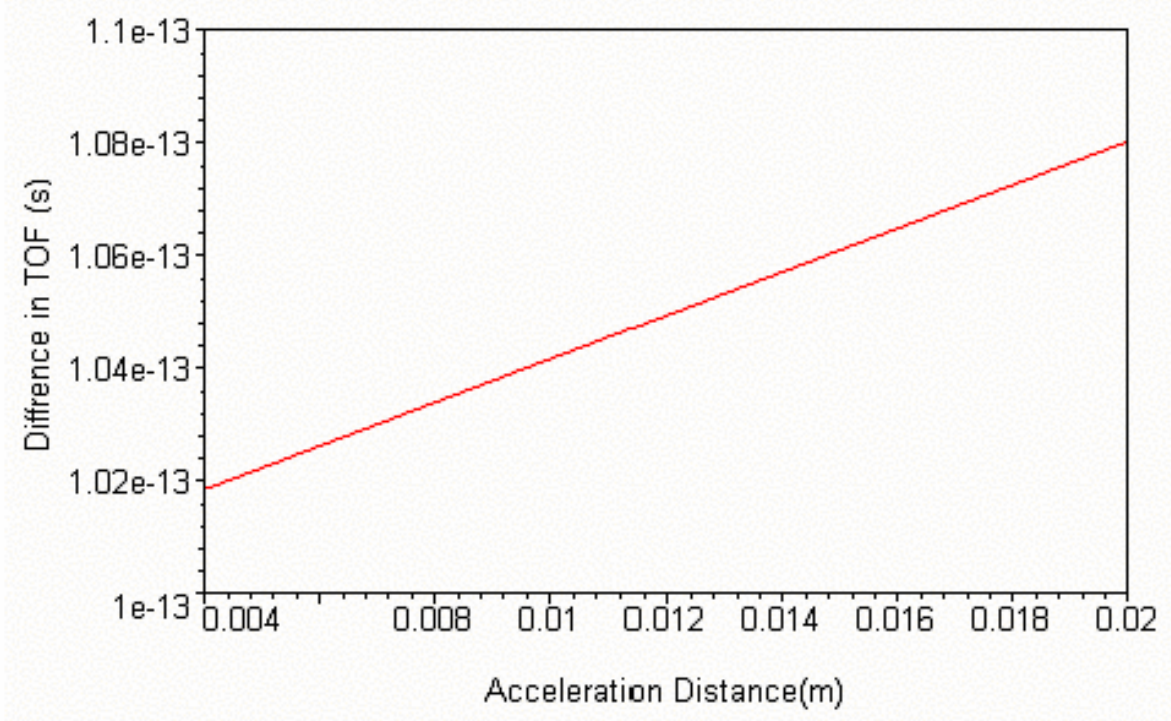

Figure 5. $\left(\mathrm{T}_{\text {rel }}-\mathrm{T}_{\text {cla }}\right)$ vs. free flight distance $\left(\mathrm{S}_{1}\right)$

The graph in figure 5 is plotted in the range of acceleration distance, $S_{1}$ from $0.4 \mathrm{~cm}$ to $2 \mathrm{~cm}$, whereas the typical value for $S_{l}$ is 1.23 centimeters. The time difference is still seen to be of the order $10^{-13}$ seconds.

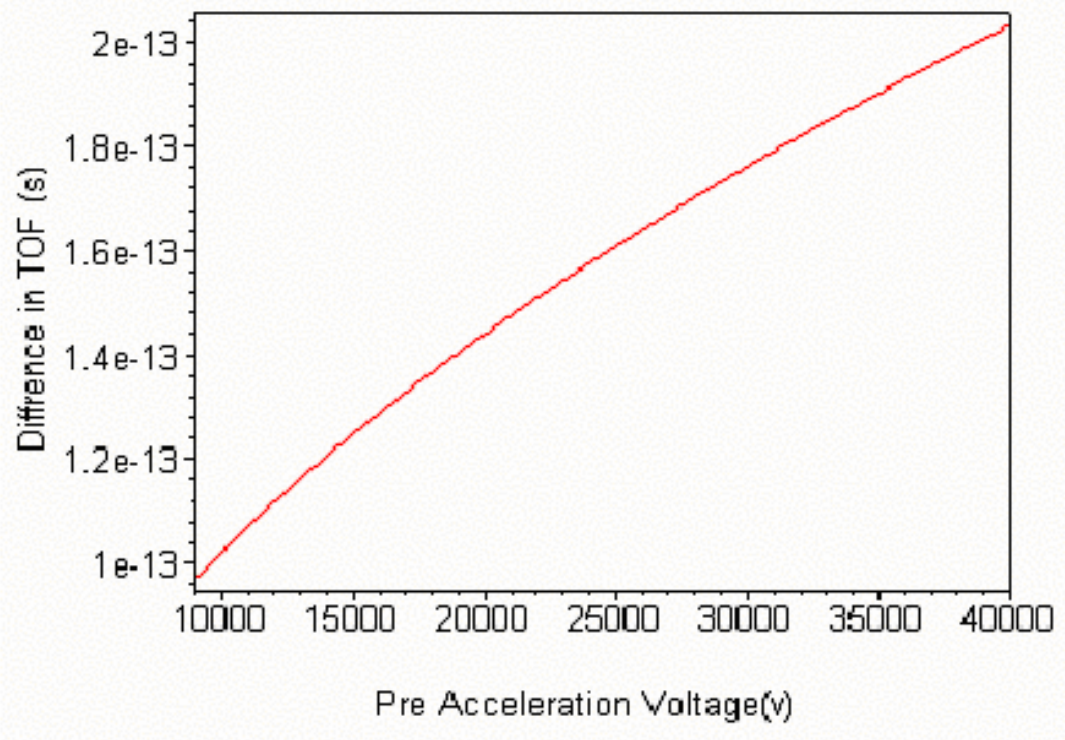

Figure 6. $\left(\mathrm{T}_{\text {rel }}-\mathrm{T}_{\text {cla }}\right)$ Vs. free flight distance $\left(\mathrm{V}_{1}\right)$ 
The graph in figure 6 is plotted for acceleration voltage $V_{l}$, from 9000 volts to 40000 volts. The time difference is still seen to be of the order $10^{-13}$ seconds. Since the time resolution of the PDMS at the department of physics is 1 nanosecond, this difference is very much negligible.

\section{RESULTS AND DISCUSSION}

Corresponding $\mathrm{m} / \mathrm{q}$ for a given TOF and spectrometer parameters could be calculated by applying classical equation (1) or relativistic equation (14). But it was seen that the difference between the two results is of order $10^{-5}$ a.m.u. per electron charge. Further more, the instrumental errors suppress the relativistic corrections. Hence it could be concluded that the relativistic effects are negligible.

Also, it was seen that even when spectrometer parameters such as pre acceleration voltage $\left(V_{l}\right)$, pre acceleration distance $\left(S_{1}\right)$ and free flight distance $\left(S_{2}\right)$ was increased to promote relativistic effects, still the difference between the two values of the TOF was of the order $10^{-13}$ seconds. Therefore it can be concluded that for the current type of PDMS, relativistic effects are negligible and classical TOF equation (1) is sufficient for calculating $m / q$.

\section{REFERENCES}

1. Abeywarne, U.K. (1999). Initial Energy Distributions of Secondary Ions in Electronic Sputtering (Thesis). University of Colombo, Sri Lanka.

2. Daya, D.N.B. (2003) (Private Communication)

3. Emmen, A. (1998). NAG software to be embedded in Maple mathematical system. www.hoise.com/primeur/00/articles/AE-PR-09-98-23.html

4. Kenneth Ashdown. (2003). About NAG. www.nag.co.uk/about_nag.asp

5. Press, W.H., Vellering W.T., Teukolsky, S.A., Flannery, B.P. (1986) Numerical Recipes in Fortran. pp. 281-287. Cambridge University Press. (Cambridge)

6. Widdiyaseksra, S., Hakansson, P., Sundqvist, B.U.R. (1987). A method of measurements of the initial axial velocity distribution of secondary ions in electronic sputtering of organic molecules. Internal Report TSL-ISV:4 Uppsala University (September 1987) pp.1-10. 